\title{
CONVUlsiones EN EL PRIMER TRIMESTRE DE LA VIDA
}

\author{
(Estudio de 36 njn̄os)
}

\author{
Dr. MARIANO LATORRE y Dra. JESSIE MANHOOD
}

\begin{abstract}
Cátedra de Pediatria Prof. A. Arizlia, Hospital de Niños "Luis Calvo Mackənna”.
\end{abstract}
La aparición de crisis convulsivas en un niño menor de tres meses y en especial del recién nacido, mueve a discutir siempre las más sombrías hipótesis respecto al pronóstico inmediato, y al tardío. La observación de algunos casos cuya evolución fué satisfactoria movió a analizar el material clínico estudiado en el Hospital "Luis Calvo Mackenna", con el fin de investigar el desarrollo psicomotor de estos niños, y eventualmente relacionar algunos signos y sintomas que podian orien. tar el pronóstico.

Se consideró de interés estudiar la evolución del sindrome convulsivo en este periodo de la vida, ya que el Sistema Nervioso se encuentra en estado de inmadurez, y las lesiones provocadas pueden ser irreversibles, con la detención consiguiente del desarrollo psicomotor.

Se seleccionaron niños que reunieran las características siguientes:

$1^{\circ}$ Que fueran menores de tres meses de edad.

$2^{\circ}$ Que hubieran presentado convulsiones, éstas debian ser con pérdida del conocimiento, y generalizadas, o que comprometieran como mínimo dos extremidades y cara. No entraron en la serie níños con sacudidas clónicas aisladas, ya que se ven frecuentemente en recién na. cidos normales.

$3^{\circ}$ Se exigió un plazo mínimo de control de seis meses después del alta, para apreciar el estado del desarrollo psicomotor.

4! Todos los niños habian sido hospitalizados.

Para buscar estos casos se revisaron las fichas clínicas de niños cuyo diagnóstico permitiera sospechar la presencia de convulsiones, sin hacer selección respecto a etiologia. Se estudiaron entonces Epilepsia (Gran Mal). Meningitis Purulenta, Espásticos, Encefalitis, Hemorragia Intracraneana, Hematoma Subdural, Ictericia Nuclear; y además, las fichas dẹl Centro de Prematuros correspondientes a los niños que ingresaron a dicho Centro entre los años 1956-1957, comprendiendo tanto Ios que habían sido dados de alta como los fallecidos.

En el Centro de Prematuros se archivan las observaciones clínicas durante un año para el control del niño en la Policlínica del Centro, pasando después la ficha a Estadística Central. Además, hay que agregar que se archivan por número y no por díagnóstico, por lo que el estudio no se hizo en forma dirigida como en Estadística Central, sino que se revisó la totalidad de las fichas.

Una vez terminado el estudio se vió que la revisión había comprendido desde el año 1943 hasta 1958 inclusive, encontrándose los casos que se detallan a continuación:

CUADRO No 1

TOTAL DE CASOS AGRUPADOS POR DLAGNOSTICO

\begin{tabular}{l}
\hline \\
Eran mal $\ldots \ldots \ldots \ldots \ldots \ldots \ldots \ldots \ldots \ldots \ldots \ldots \ldots$ \\
Menin $\ldots \ldots \ldots \ldots$
\end{tabular}

TOTAL

1,119

Entre todos estos casos se encontró un total de 48 niños aue presentaron convulsiones durante los 3 primeros meses de la vida. 12 de ellos se eliminaron de la serie, 9 de ellos no se encontraron en el domicilio que figuraba en la ficha clínica, se ignoraba, por lo tanto, su evolución posterior, dos habian fallecido en el hogar, según informe de la Enfermera Sanitaria; 
$\mathrm{y}$ finalmente 1 por tener información que se consideró incompleta.

Quedó un total de 36 niños que se distribuyen por diagnóstico en la forma siguiente:

\section{CUADRO NQ 2}

NIYO3 QUE PRESENTARON CONVUISIONES EN ET. PRIMER TRIMESTRE DE LA VWA

\begin{tabular}{|c|c|}
\hline & No de miños \\
\hline 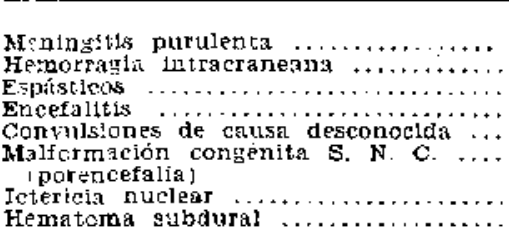 & $\begin{array}{r}14 \\
7 \\
6 \\
4 \\
2 \\
1 \\
1 \\
1\end{array}$ \\
\hline TOTAL & 36 \\
\hline
\end{tabular}

A continuación viene un cuadro resumen de todos los casos; y en las páginas siguientes, el estudio resumido de cada uno de ellos, quedando agrupados por diagnóstico 1 cuadros 3,4 y 5 ).

\section{MENINGITIS PURULENTA}

El análisis de este grupo muestra que el pronóstico de 1a Meningitis Purulenta en el menor de 3 meses sigue siendo un problema por resolver, aún en la era de los antibióticos. En el grupo de niños estudiados, de 14 lactantes 7 fallecen, 3 quedan con secuelas graves, y solamente 4 se recuperan desarrollándose normalmente.

De acuerdo con una publicación de la Dra. Hattie Alexander ${ }^{1}$, resumen de lo observado en 10 años, la alta mortalidad y las secuelas son problemas universa- les, y según su experiencia no se debe tanto a la menor edad, como a la dificul. tad para hacer el diagnóstio precoz en este grupo.

\section{HEMORRAGIA INTRACRANEANA}

En esta serie de niños 2 fallecen, siendo ambos prematuros. 1 de ellos presenta el cuadro que se describe como caracteristico de la hemorragia masiva $\mathrm{y}$ difusa. El otro, que fallece por una causa extra craneana, muestra una lesión en plena zona Rolándica, que a no dudar habría dejado una grave secuela motora.

El segundo grupo está constituido por 5 lactantes que inician las convulsiones en los primeros 5 días de la vida. Llama la atención que en todos ellos existe distocia, y aparte de la distocia en 4 de los 5 se aplicaron manjobras obstétricas forceps) y hubo además asfixia. Aun cuando este último signo no está especificado en cuanto a duración; es de suponer que excedió a lo que se ve habitualmente, por cuanto quedó consignado en el boletín (cuadros $\overline{6}, 7$ y 8 ).

Tratando de comparar algunos signos que presentaron estos niños en el momento de iniciar las convulsiones, con el fin de orientar el pronóstico; se aprecia que los signos han sido semejantes, inclusive la edad de iniciación de las convulsiones, de tal modo que en ese momento habría sido imposible pronunciarse sobre el pronóstico. Dos de los actualmente normales, han nacido en un parto distócico, con aplicación de forceps y asfixia. Los otros también han nacido en un parto distócico, dos de ellos con aplicación de forceps $y$ asfixia $y$ el tercero en un periodo expulsivo rápido sin embargo la evolución ha sido muy desfavorable.

CUADRO NO 3

CUADRO RESUMEN DE LOS 36 NLNOS RSTUDIADOS

\begin{tabular}{|c|c|c|c|c|c|}
\hline Dingnostico & $\begin{array}{l}\text { Ne total } \\
\text { de cascs }\end{array}$ & $\begin{array}{l}\text { No de } \\
\text { fallecldos }\end{array}$ & $\begin{array}{c}\text { Evolucloj } \\
\text { destavorable }\end{array}$ & $\begin{array}{l}\mid \\
\vdots \\
1 \\
1\end{array}$ & $\begin{array}{c}\text { Eroluctón } \\
\text { buaena }\end{array}$ \\
\hline 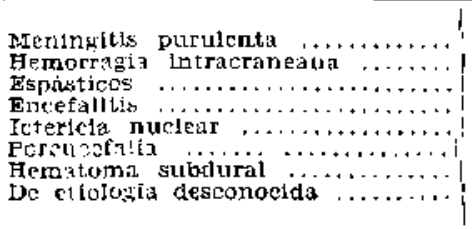 & $\begin{array}{r}14 \\
7 \\
6 \\
4 \\
1 \\
1 \\
1 \\
2\end{array}$ & $\begin{array}{c}1 \\
\vdots \\
\vdots \\
\vdots \\
\vdots \\
\vdots \\
\vdots \\
\vdots\end{array}$ & $\begin{array}{l}3 \\
3 \\
5 \\
2 \\
\end{array}$ & $\begin{array}{c}1 \\
\vdots \\
\vdots \\
1 \\
\vdots \\
1 \\
1 \\
1 \\
1\end{array}$ & $\begin{array}{c}4 \\
\mathbf{2} \\
1 \\
1 \\
2\end{array}$ \\
\hline
\end{tabular}


CDADRO Nó 4

MENINGITIS PURDLENTA: NINOS FALIECIDOS

\begin{tabular}{|c|c|c|c|c|}
\hline Caso Ne & Parto & p s tolos i c a & $\begin{array}{c}\text { Euad } \\
\text { Inlclactón } \\
\text { convulstones }\end{array}$ & $\begin{array}{l}\text { Pdad } \\
\text { falleclmiento }\end{array}$ \\
\hline 0 & Normal & $\begin{array}{l}\text { Manclias purpurieas de la plel, exudado purulento } \\
\text { de las monimges, otitis purilenta bilateral. }\end{array}$ & 12 dias & 12 días \\
\hline 10 & Cesátea & $\begin{array}{l}\text { Exudado purulento que cubre convexidad cerebro, } \\
\text { cerebelo, protuberincla. bulbo. outs media puru- } \\
\text { lenta. Foco3 supurados pulmonares. }\end{array}$ & 20 dios & 20 días \\
\hline 11 & Normal & $\begin{array}{l}\text { Exudado purulento de convexldad, base dal cerebelo } \\
\text { y ventricilos. Focos bronconeumonleos. }\end{array}$ & $\begin{array}{l}2 \text { mescs } \\
13 \text { dlas }\end{array}$ & $\begin{array}{l}2 \text { meses } \\
17 \text { dias }\end{array}$ \\
\hline 12 & Normai & $\begin{array}{l}\text { Exudedo purulento en convexidad, base, ventrichl- } \\
\text { los, gule se thsinua ipor cl canal raquideo, cubrlendo } \\
\text { la métula. }\end{array}$ & $\begin{array}{l}1 \text { mes } \\
7 \text { dias }\end{array}$ & $\begin{aligned} 1 & \text { mes } \\
12 & \text { dias }\end{aligned}$ \\
\hline 13 & Norma] & 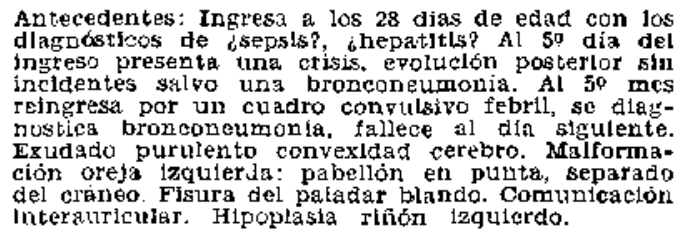 & $\begin{array}{l}1 \text { mos } \\
3 \text { dias }\end{array}$ & 5 ineses \\
\hline 14 & Normal & 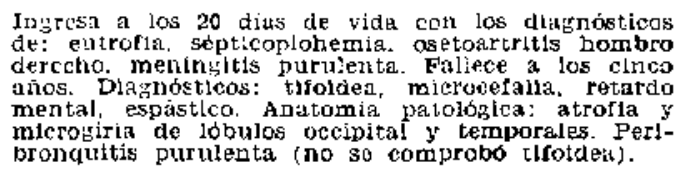 & 21 dias & 5 arios \\
\hline 15 & Normal & 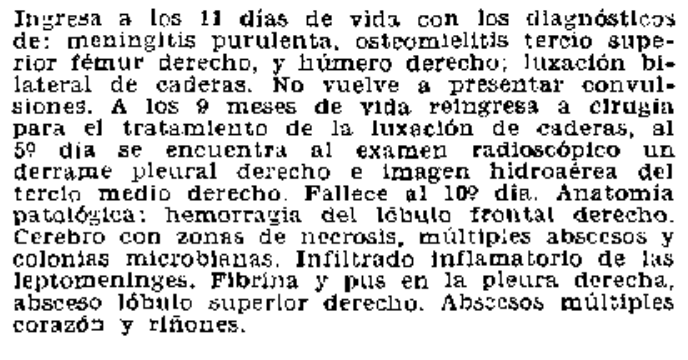 & 11 días & i0 meses \\
\hline
\end{tabular}

\section{ESPÁTICOS}

Se agrupan bajo este diagnóstico a los Espásticos cuya causa fué probablemente prenatal. La sintomatología neurológica posterior corresponde a este cuadro clínico, y porque en el parto no hubo ningún sintoma que hiciera sospechar la presencia de una hemorragia intracraneana.

En el momento del parto, aparte del signo común que fueron las convulsiones, no hubo otro detalle que hiciera sospechar la evolución posterior, uno de los niños era prematuro, en uno hubo asfixia azul, en un caso se practicó operación Cesárea, en uno el período expulsivo fué rápido y en dos el parto fue normal. (Cuadro $\mathrm{N} \div 9$ ).

Este hallazgo esta de acuerdo con to expresado en otras publicaciones. Si se recuerda que se seleccionaron seis en un total de noventa y nueve Espásticos, y solamente estos seis presentaron convulsiones en el primer trimestre de la vida, da la impresión que este signo no es de gran valor para el diagnóstico. 
CUADRO NQ 5

MENINGITIS PURULENTA: EVOLDCION POSTERIOR

\begin{tabular}{|c|c|c|c|c|}
\hline Caso NQ & Patto & $E \nabla 014016 n$ & $\begin{array}{c}\text { Ediad } \\
\text { 1ntclación } \\
\text { convulstones }\end{array}$ & $\begin{array}{l}\text { Tlempo } \\
\text { eroluckón }\end{array}$ \\
\hline 7 & $\begin{array}{l}\text { Periodo ex- } \\
\text { pulsivo rá } \\
\text { pido (5 mi- } \\
\text { butos) }\end{array}$ & $\begin{array}{l}\text { Mala. Bla contacto con el amblente. Tendencla aI } \\
\text { oplstotonos. Hidrocefalia detenlda post menlngitis }\end{array}$ & 5. días & 6 mcses \\
\hline & & 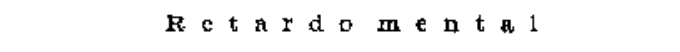 & & \\
\hline 6 & Normal & $\begin{array}{l}\text { Apreciaclón clinica: no ge practicaron tesle (vive } \\
\text { fuera de la ciudad). }\end{array}$ & $\begin{array}{l}1 \text { mes } \\
7 \text { dias }\end{array}$ & 9 anos \\
\hline 5 & $\begin{array}{l}\text { Prematuro } \\
(1,600 \text { ir. }\end{array}$ & $\begin{array}{l}\text { C. I. de } 71 \text { en test de Goopenough, } 74 \text { Binet redu- } \\
\text { cldo. E. E. G.: alteración difusa cortlco subcortical. }\end{array}$ & $\begin{aligned} 2 & \text { meses } \\
27 & \text { dias }\end{aligned}$ & 7 axos \\
\hline & & B & & \\
\hline 4 & Nornal & Desarrollo zormal. & $\begin{array}{l}1 \text { mes } \\
22 \text { djas }\end{array}$ & 2 aร̌os \\
\hline 3 & Normal & Desarrollo tormal, & 13 dias & $\begin{array}{l}3 \text { afios } \\
8 \text { meses }\end{array}$ \\
\hline 2 & $\begin{array}{l}\text { Prematuro } \\
(2.000 \text { g. }\end{array}$ & Desarrollo normal. & $\begin{array}{l}2 \text { meses } \\
2 \text { dias }\end{array}$ & 6 afios \\
\hline I & Normal & Desartollo normal. & 12 mes & $\begin{array}{l}1 \text { afio } \\
7 \text { meses }\end{array}$ \\
\hline
\end{tabular}

CUADRO No 6

HEMORRAOIA INTRACRANEANA: FALLECLOS

\begin{tabular}{|c|c|c|c|c|}
\hline Caso No & Parto & patolog $1 \mathrm{ca}$ & $\begin{array}{l}\text { Fdad } \\
\text { inlckación } \\
\text { convulsiones }\end{array}$ & $\begin{array}{c}\text { Elad } \\
\text { falleclmiento }\end{array}$ \\
\hline 24 & $\begin{array}{l}\text { Prematuro } \\
\text { fl.700 g.l } \\
\text { Periodo ex- } \\
\text { puls!vo rá- } \\
\text { pldo }\end{array}$ & $\begin{array}{l}\text { Gran cantldad de cóagulos en el créneo, mais abun- } \\
\text { dantes en el hemisferio derecho. } \\
\text { Rifiones lobulados. }\end{array}$ & $\begin{array}{l}13 \text { horas } \\
\text { de vida }\end{array}$ & 6 dias \\
\hline 23 & $\begin{array}{l}\text { Prematuro } \\
(1.940 \text { (s.) }\end{array}$ & 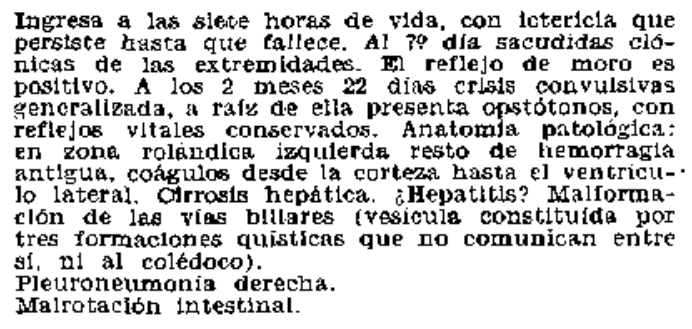 & $\begin{array}{l}2 \text { meses } \\
22 \text { días }\end{array}$ & $\begin{array}{l}3 \text { meses } \\
24 \text { dias }\end{array}$ \\
\hline
\end{tabular}


CDADRO NG 7

HEMORRAGLA INTRACRANEANA: EVOLUCION POSTERTOR

\begin{tabular}{|c|c|c|c|c|}
\hline Caso ND & Parto & Eroluctón posterior desfavorable & $\begin{array}{l}\text { Edad } \\
\text { Iniciaclón } \\
\text { convulsioyes }\end{array}$ & $\begin{array}{l}\text { Tlempo } \\
\text { evolucion }\end{array}$ \\
\hline 22 & $\begin{array}{l}\text { Fórceps } \\
\text { ¿Asrixin? }\end{array}$ & 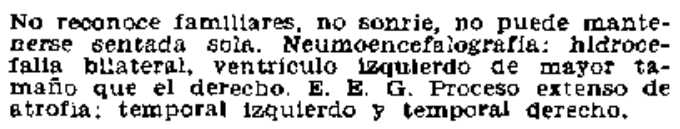 & $\begin{array}{l}\text { 3er dia } \\
\text { de rida }\end{array}$ & 4 anos \\
\hline $2 d$ & $\begin{array}{l}\text { Forceps } \\
\text { Ast } 1 \times 1 a\end{array}$ & $\begin{array}{l}\text { Ausencla del lenumaje, no deambula sola. marcha } \\
\text { espastica. Stgnos piramidales positivos, Retardo men- } \\
\text { tal que no se pudo avaluar. }\end{array}$ & $\begin{array}{l}\text { ler. dis } \\
\text { de vida }\end{array}$ & 5 añas \\
\hline 26 & $\begin{array}{l}\text { Periodo } \\
\text { expulsityo } \\
\text { répido }\end{array}$ & 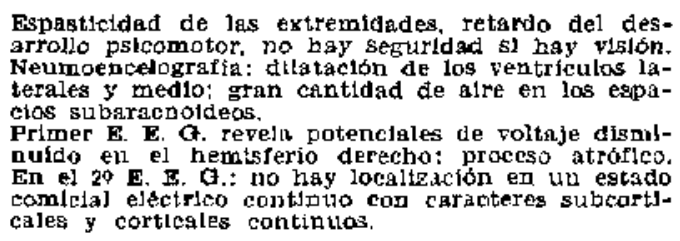 & $\begin{array}{l}5 \text { disa } \\
d e^{4}+d a\end{array}$ & $\begin{array}{ll}2 & \text { añas } \\
11 & \text { meses }\end{array}$ \\
\hline
\end{tabular}

CUADRO NQ a

HEMORRAGIA INTRACRANEANA: EVOLUCION POSTERIOR

\begin{tabular}{|c|c|c|c|c|}
\hline Caso IY & Parto & Ev & $\begin{array}{l}\text { Ejad } \\
\text { inlclactón } \\
\text { convulsiones }\end{array}$ & $\begin{array}{l}\text { Thampo } \\
\text { evolución }\end{array}$ \\
\hline 17 & $\begin{array}{l}\text { Fórceps } \\
\text { Asfixla }\end{array}$ & $\begin{array}{l}\text { Se sentó sin apoyo a los } 2 \text { meses, primeros pasos y } \\
\text { palabras al año, controló esfinteres antes de los dos } \\
\text { afos. }\end{array}$ & $\begin{array}{l}\text { do día } \\
\text { de vida }\end{array}$ & $\begin{array}{l}4 \text { gños } \\
4 \text { meses }\end{array}$ \\
\hline 16 & $\begin{array}{l}\text { Embarazo } \\
\text { prodongado. } \\
\text { Parto de } \\
\text { nalgas. } \\
\text { Fórceps. } \\
\text { Astlaia. }\end{array}$ & $\begin{array}{l}\text { Primeras paiabras a los } 10 \text { meses, primeros pasos a } \\
\text { los a meses, Al año podia mimenterse por si misno. } \\
\text { Controló estinteres antes de los } 2 \text { años. }\end{array}$ & $\begin{array}{l}\text { ler. día } \\
\text { de vida }\end{array}$ & 3 intas \\
\hline
\end{tabular}

\section{ENCEFALITIS}

Del grupo de cuatro Encefalitis, uno de ellos fallece, comprobándose la presencia de exudados perivascular en el examen microscópico de la corteza cerebrïl.

Aun cuando el diagnóstico clínico puede merecer dudas en los otros 3, por cuanto no fué posible comprobar la presencia de virus, se puede estar razonablemente seguro por el cuadro clínico y la presencia de secuelas. Es sabido que el compromiso secundario del Sistema Nervioso Central en el lactante da un cuadro semejante, pero el nin̄o emerge de su in* consciencia absolutamente indemne.

(Cuadro No 10 ).

\section{ICTERICLA NUCLEAR; PORENCEFALIA Y HEMATOMA SUBDURAL}

Ictericia Nuclear. En la práctica diaria se hace hincapié en la presencia de convulsiones como uno de los índices de impregnación nuclear. La observación de los casos estudiados habia hecho dudar de esta premisa; y en esta serie, uno solo de nueve lactantes presentó convulsiones.

Porencefalia. Corresponde incluir a este lactante dentro de las anormalidades del desarrollo del Sistema Nervioso Central.

Habitualmente este grupo de enfermedades produce convulsiones después del sexto mes de la vida, sin embargo en el único caso de esta serie se iniciaron en el 
CUADRO No 9

E S P A T I COS

\begin{tabular}{|c|c|c|c|c|}
\hline Caso No & Parto & Fat 1 ectal o & $\begin{array}{c}\text { Ediad } \\
\text { Iniciación } \\
\text { convulstonez }\end{array}$ & Edad \\
\hline 25 & $\begin{array}{l}\text { Asfixia azul } \\
\text { islntamas } \\
\text { de aborto } \\
\text { 5o mes }\end{array}$ & 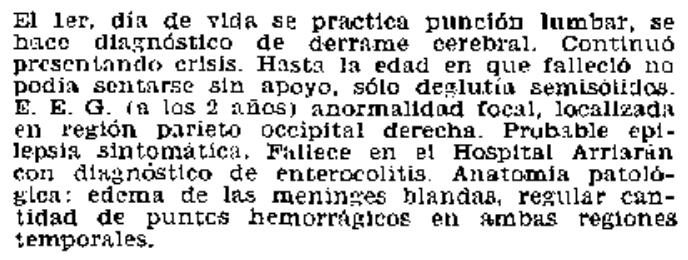 & $\begin{array}{l}\text { ler. dia } \\
\text { de vida }\end{array}$ & 4 anos \\
\hline Caso Nọ & Parto & Evolucion destavorabla & $\begin{array}{c}\text { Edad } \\
\text { iniclatión } \\
\text { convulsiones }\end{array}$ & $\begin{array}{l}\text { Tlerapo } \\
\text { evolucion }\end{array}$ \\
\hline 20 & Cesánen & $\begin{array}{l}\text { Todos los atributus de un daño cerebral congénito, } \\
\text { con cspiastldand. }\end{array}$ & $\begin{array}{l}2 \% \text { dia } \\
\text { de vida }\end{array}$ & $\begin{array}{l}2 \text { años } \\
4 \text { meses }\end{array}$ \\
\hline 10 & $\begin{array}{l}\text { Periodo } \\
\text { expulsivo } \\
\text { rápldo }\end{array}$ & $\begin{array}{l}\text { Ha continuado presentrando crisis, se aprecin exis- } \\
\text { tencia de espasilcidiad } y \text { retardo mental. }\end{array}$ & $\begin{array}{l}\text { ler. din } \\
\text { de pida }\end{array}$ & $8 \operatorname{angs}$ \\
\hline IB & $\begin{array}{l}\text { Parto } \\
\text { normal }\end{array}$ & $\begin{array}{l}\text { Froite atgunas pinlabris, rfglder generallzada, refle- } \\
\text { jos profundos exnltados. }\end{array}$ & 1 mes & 4 คกตร \\
\hline 27 & Normal & $\begin{array}{l}\text { Fopasticidad generallaida, movimientas catereotipa- } \\
\text { dos sin fin aparente de ias extremldartis. Bibinsky } \\
\text { posttluo bilateral. }\end{array}$ & $\begin{array}{l}\text { 3er. din } \\
\text { de vldat }\end{array}$ & $\begin{array}{l}1 \text { iño } \\
9 \text { meses }\end{array}$ \\
\hline 28 & $\begin{array}{l}\text { Prematuro } \\
(2.000(\mathrm{~L})\end{array}$ & 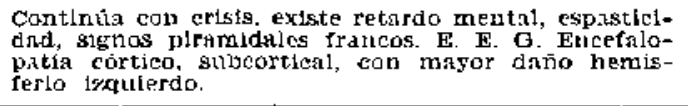 & $\begin{array}{l}20 \text { dia } \\
\text { de vlat }\end{array}$ & 12 axas \\
\hline
\end{tabular}

CUADRO No 10

E N C E F A L I T I

\begin{tabular}{|c|c|c|c|c|}
\hline Criso NQ & Parto & F A 1 l e c I d o & $\begin{array}{c}\text { Edad } \\
\text { Intelacion } \\
\text { convulsiones }\end{array}$ & $\begin{array}{c}\text { Edrui } \\
\text { rallectmiento }\end{array}$ \\
\hline $\mathbf{B}$ & Normal & 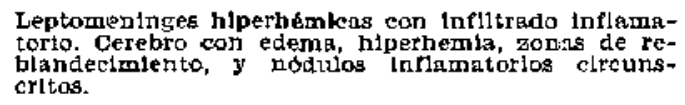 & $\begin{array}{l}2 \text { meses } \\
14 \text { djas }\end{array}$ & $\begin{array}{l}2 \text { meses } \\
21 \text { aijs }\end{array}$ \\
\hline Caso Nọ & Pacto & E & $\begin{array}{l}\text { Edad } \\
\text { inicigcion } \\
\text { convulsiones }\end{array}$ & $\begin{array}{l}\text { Tlempo } \\
\text { evoluclón }\end{array}$ \\
\hline 29 & Normal & $\begin{array}{l}\text { No sostiene la cabera, no se sienta sin apoyo. crl- } \\
\text { sis de tipo diencefílico. }\end{array}$ & 1 mes & $\begin{array}{l}2 \text { años } \\
6 \text { meses }\end{array}$ \\
\hline 30 & Normal & Marcha qacilante, continuia con crisis. & 28 mes ou & 3 años \\
\hline & & Evolue 10 n 2 a vorable & & \\
\hline 31 & Normal & $\begin{array}{l}\text { No volvió à presentar crisis primeros pasoe y pri- } \\
\text { meras palabras al año, controlo esfímteres antes de } \\
\text { los tres anos. }\end{array}$ & 2 meser & 4 afios \\
\hline
\end{tabular}


CUADRO No 11

CASOS DE ICTERTCLA NUCLEAR, PORENCEFALIA $Y$ HEMatoMA SUBdURAL

\begin{tabular}{|c|c|c|c|c|}
\hline Caso Nọ & Parto & E $\nabla \circ I U<16 \mathbf{n}$ & $\begin{array}{c}\text { Edad } \\
\text { 1miclación } \\
\text { convuls lones }\end{array}$ & $\frac{\text { Edad }}{\text { fallecimien to }}$ \\
\hline & & Ietericia nuclear & & \\
\hline 34 & Normal & $\begin{array}{l}\text { Antecedentes: madre Rh (-): niño kh ( } i) \text {. Ingresa } \\
\text { a las } 75 \text { horas de vida, no se hibo recambio. Anato- } \\
\text { mia patológlca: coloraclón amarilir de los núcleos } \\
\text { centrates del cerebro. } \\
\text { Hemprragjas subpleurales. }\end{array}$ & $\begin{array}{l}\text { 20 aia } \\
\text { de vida }\end{array}$ & $49 \varphi \mathrm{dia}$ \\
\hline Caso No & Parto & Porencefalia & $\begin{array}{c}\text { Eaad } \\
\text { Inlciacion } \\
\text { convustones }\end{array}$ & $\begin{array}{l}\text { Tlempo } \\
\text { evolución }\end{array}$ \\
\hline \multirow[t]{2}{*}{33} & Normal & 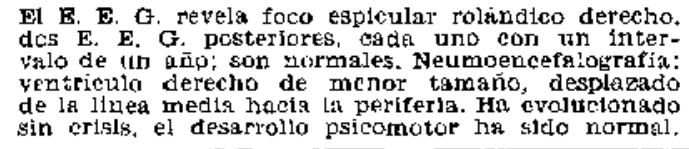 & $\begin{array}{l}\text { 2a dia } \\
\text { de vlaa }\end{array}$ & $\begin{array}{l}2 \text { Rfios } \\
9 \text { meses }\end{array}$ \\
\hline & & Hematoma subdural & & \\
\hline 32 & $\begin{array}{l}\text { Prematuro } \\
(2.000 \mathrm{~g} .) \\
\text { Forceps }\end{array}$ & 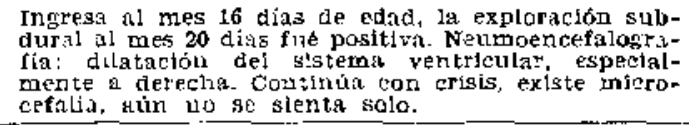 & $\begin{array}{l}\text { zo dia } \\
\text { de vida }\end{array}$ & $\begin{array}{l}2 \text { anos } \\
1 \text { mes }\end{array}$ \\
\hline
\end{tabular}

CUADRO NO 12

CONVULSIONES DE CAUBA DESCONOCIDA

\begin{tabular}{|c|c|c|c|c|}
\hline Caso Ne & Parto & $E v o l u c i d n$ & $\begin{array}{c}\text { Ejad } \\
\text { inleiación } \\
\text { convulsiones }\end{array}$ & $\begin{array}{l}\text { Tiempo } \\
\text { evalueión }\end{array}$ \\
\hline 35 & Fórceps & $\begin{array}{l}\text { Presenta dog crisis con oche dins de lntervalo, exa- } \\
\text { men fisloo negativo. } \\
\text { E. F, G. Iue normal. } \\
\text { Evoluciona sin crisis, desarroilo palcomotor ha sldo } \\
\text { normal. }\end{array}$ & $\begin{array}{l}\text { I mes } \\
2 \text { dias }\end{array}$ & $\begin{array}{l}2 \text { affos } \\
1 \text { mes }\end{array}$ \\
\hline 38 & Normal & 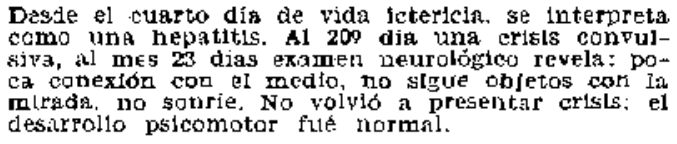 & 20 dias & $\begin{array}{l}1 \text { año } \\
4 \text { meses }\end{array}$ \\
\hline
\end{tabular}

segundo día de la vida. La persistencia de este signo junto a un desarrollo reiativa. mente normal, indujo a un estudio completo; verificándose en la Neumoencéfalografía (practicada en Neurocirugía) la presencia de una cavidad quística en el Hemisferio Derecho, comunicada con el ventriculo es decir, una Porencefalia Vera. Llama la atención que un defecto tan grosero del Sistema Nervioso Central sea compatible con un desarrollo normal, tanto psiquico como motor. (Cuadro $\mathrm{N}^{\prime} 11$ ).

Hematoma subdural. También este caso único plantea un problema semejante al de Ia Ictericia Nuclear. El hallazgo de convulsiones, aunque descrito entre los signos del Hematoma Subdural, es un hecho que no se observa frecuentemente. (En los 16 casos estudiados 1 solo lo presentaba).
Este lactante hizo la evolución típica del Hematoma Subdural no operado, que se tradujo en microcefalia y daño psicomotor.

Convulsiones de etiología desconocida.

Se han agrupado bajo este título 2 lactantes que presentan convulsiones, 1 a los 20 días de edad y el otro a $\operatorname{los} 32$ días; en los cuales la investigación clínica y el desarrollo posterior no han permitido establecer etiologia. (Cuadro $N^{\prime \prime} 12$ ).

El desarrollo osicomotor normal, si bien no permite eliminar en forma absoluta la posibilidad de un daño cerebral congénito lo hace poco verosímil.

Aunque la epilepsia puede iniciarse en esta edad, en general no deja espacio intercrisis tan largos, ( 1 año 4 meses, 1 año 
1 mes) además la incidencia de la Epilepsia como causa de convulsíones en el primer trimestre es de un $0.8 \%$.

Queda por considerar un factor que aún no se ha descrito en nuestro medio. La deficiencia de vitamina B6 fué descrita como causa de convulsiones en lactantes alimentados artificialmente en E.E. U.U. con un solo tipo de alimento: la leche S.M.A. (Similar Mother's Aliment) en 1950 .

\section{RESUMEN}

El objetivo de la presente publicación fué analizar un grupo de enfermos que iniciaron un sindrome convulsivo en los tres primeros meses de la vida; estudiar el proceso etiológico, y relacionarlo con el pronóstico.

Aun cuando el número de niños analizados no permite generalizar los hallazgos de este estudio a todos los menores de tres meses de edad, se observa que en cuanto a etiología se ajusta a lo descrito en otras publicaciones. Los factores etiológicos encontrados en esta serie en orden de frecuencia son:

1. Infecciones Microbianas del S.N.C. (Meningitis Purulenta).

2. Hemorragia Intracraneana.

3. Espásticos.

4. Encefalitis.

5. Convulsiones de Etiología Desconocida.

6. Malformaciones Congénitas del $\mathrm{S}$. N.C.

7. Ictericia Nuclear.

8. Hematoma Subdural.

El signo convulsiones en el primer trimestre de la vida significa pronóstico grave y exige una investigación cuidadosa de la etiologia, dependiendo de ella la evolución posterior. Las convulsiones secundarias a Meningitis Purulenta y a Hemorragia Intracraneana en este grupo de niños son más graves que las debidas a otra etiología.

\section{SUMMARY}

CONVULSIVE DISORDER IN THE FIRST THREE MONTHS OF AGE.

The authors presents a group of 36 children observed in the Children's Hospital "Luis Calvo Mackenna", Santiago, that have presented convulsive disorders in the first three months of age. Etiological factors and prognosis are analyzed.

Based in their experience, convulsive disorder in the first trimester of life has a serious prognosis, especially if purulent meningitis or cerebral hemorrage is present, where a thorougly investigation must be done.

\section{BIBLIOGRAFÍA}

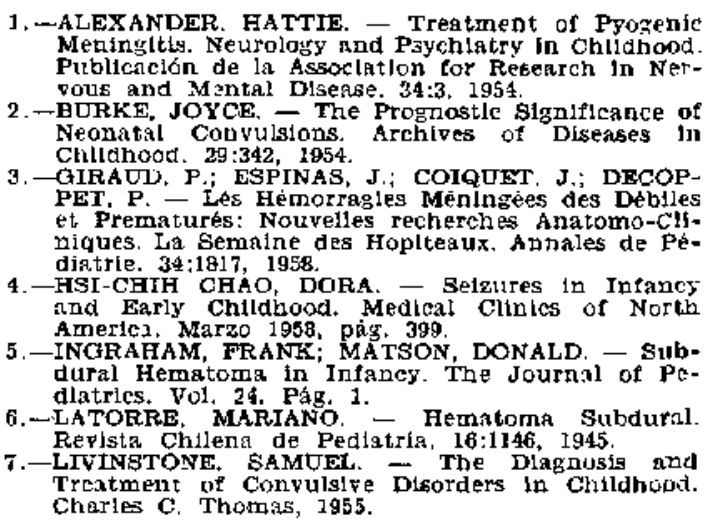

\section{DISCUSIóN}

Dr. Marin Sefuifueda: Expresa que en el Hospital Sin Juan de Dios han observado que las convulsiones mis frecuentes del primer trimestre de vida cotresponden a las hemotragios intracraneanas.

Dr. Murio Gonzúlez: Manifiesta que en 479 laclantes con toxicosis ellos han observado que 72 thvieron convlusioncs, y por lo tanto estima que debe tenerse en cuenta tambión a la desthidratación aguda como causa de este sindrome.

Dr. Ricarda Otea: Considera que la hemorrngix intrictaneani es la causa mis importante de convulsiones en los niños menores de 15 ó 30 dias de edad. Despuis incluye la meningitis. Mucho de los casos de roxicosis pueden cortesponder a encefalitis. L.as convulsiones por infeccion son de mejor pronóstica.

Prof Wicdarhold: Agrega que mucho de las convulsioncs de las toxicosis pasan con la hidratación. dehido a que co este cuadro clínico existe deshidratación general son sobrehidratación encefálica que mejota con la hidratación bicn becha.

Dr. Mariano Lasorre: Está de acuerdo que en el reciún nacido la hemortagia intracraneana juega un rol fundamental en las convulsiones. Recuerda que el trabajo presentado comprende al primer trimestre, donde no han incluido a lactantes con toxicosis. 